\title{
Pregnancy prolongation for pre-eclampsia: the obvious and the avoidable (Mini-commentary on BJOG-20-0468.R1)
}

\author{
Rossana Orabona ${ }^{1}$ and Federico Prefumo (Italy) ${ }^{1,1}$ \\ ${ }^{1}$ University of Brescia
}

August 23, 2020

The paper by Mulder et al. addresses the effect of pregnancy prolongation on maternal and fetal outcomes in women with early-onset pre-eclampsia diagnosed after 24 weeks of gestation (Mulder et al., BJOG 2020 xxxx). They report that pregnancy prolongation - from the time of pre-eclampsia diagnosis to delivery is associated with improved offspring outcome and survival, without adverse consequences on short-term maternal cardiovascular and metabolic function. The maternal findings are apparently at odds with another recent study from New York (Rosenbloom et al. Obstet Gynecol 2020;135:27-35) which observed an increased risk of maternal cardiovascular events after pregnancy, in case of an interval of more than 7 days between the diagnosis of any hypertensive disorders of pregnancy and delivery.

Some issues should be pointed out in order to avoid misunderstandings about these findings. Being Mulder et al.'s an observational study, readers cannot infer causality because women were not randomized to the length of pregnancy prolongation. Data are spread over a significant time period (from 1996 to 2017), and this aspect could be another confounder. Pre-eclampsia is a multi-organ syndrome based on chronic inflammation, oxidative stress and endothelial dysfunction leading to a persistent subclinical cardiovascular impairment and an increased risk of adverse events later in life (Sciatti et al., Eur J Prev Cardiol 2020 doi: $10.1177 / 2047487320925646$ ), similarly to what happens in cases with heart failure with preserved ejection fraction. Myocardial geometry and ejection fraction are not sensitive enough to be altered by just a few days of pregnancy prolongation, and to forecast cardiovascular consequences. Only innovative techniques such as speckle-tracking imaging may document an impairment in myocardial contractility and relaxation in former pre-eclamptics, even if ejection fraction is normal.

Pre-eclampsia is currently defined as new-onset hypertension combined with de-novo proteinuria and/or "adverse conditions" or "severe maternal/fetal complications" (Magee et al., Pregnancy Hypertens 2014;4:105145). International guidelines recommend that women with severe forms of pre-eclampsia should be delivered immediately regardless of gestational age, while an expectant management should be considered for women with non-severe pre-eclampsia before term (Magee et al., Pregnancy Hypertens 2014;4:105-145; NICE guideline no. 133, 2019). Delaying delivery is expected to benefit newborn's health, which is well exemplified by Mulder et al.'s findings. However, the fetus is often the protagonist of adverse conditions, and severe complications such as fetal growth restriction, often co-exist with early-onset pre-eclampsia, requiring longitudinal monitoring with Doppler ultrasound and cardiotocography. The timing of delivery depends on both maternal and fetal conditions. The lack of data about fetal growth and Doppler and cardiotocography findings (in cases with growth restricted babies) limits the generalisability of the results by Mulder et al. One would expect that a longer delay before delivery can be attained only in fetuses and mothers with milder clinical manifestations of disease.

Word count: 455

\section{Disclosure of interests}


The authors declare no conflicts of interest. 